\title{
Natural course of rotator cuff syndrome in a French working population
}

\section{J Bodin', C Ha², A Petit ${ }^{1,3}$, A Descatha ${ }^{4}$, M Goldberg', A Leclerc ${ }^{4}$, Y Roquelaure ${ }^{1,3}$}

${ }^{1}$ LUNAM University, University of Angers, Laboratory of Ergonomics and Epidemiology in Occupational health, Angers, France

${ }^{2}$ French Institute for Public Health Surveillance, Department of Occupational Health, Saint-Maurice, France

\author{
${ }^{3} \mathrm{CHU}$ Angers, Angers, France
}

${ }^{4}$ University of Versailles St-Quentin, UMRS 1018, Population-Based Epidemiological Cohorts' Research Platform, Villejuif, France

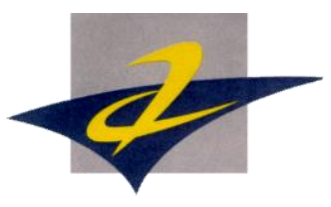




\section{Context: Rotator cuff syndrome}

- Common upper-extremity musculoskeletal disorder (MSD) in the working population

- In France (2011), shoulder disorders represented 37\% of MSD compensated as occupational diseases

- Prognosis of rotator cuff syndrome (RCS):

- In general practice: 54\% recovered after one year (Van Der Windt et al, 1996)

- In industrial and services workers: 50\% recovered after 10 months (Bonde et al, 2003) 


\section{Objectives}

- To assess the persistence of RCS in workers several years after diagnosis

- And to study associations between the persistence of RCS and personal and work-related factors and change of exposure since baseline

- Using the data of the surveillance program for MSD in the French Pays de la Loire region 


\section{Methods}

- Baseline: 2002-2005

口 Network of 83 occupational physicians (18\% of OP of the region)

\ 3,710 workers randomly selected

口 Self-administered Questionnaire

- Individual factors

- Work-related factors (work organization, biomechanical and psychosocial factors)

๑ Physical examination

- Follow-up: 2007-2010

$\checkmark$ Physical examination 


\section{Methods}

\section{- Case definition}

a Rotator cuff syndrome defined following the recommendations of the Criteria document for evaluating the work-relatedness of MSD ${ }^{1}$

Case definition 2: rotator cuff syndrome, based on symptoms and physical examination signs

\begin{tabular}{|ll|}
\hline Time rule: & $\begin{array}{l}\text { Symptoms present now or on at least } 4 \text { days during the } \\
\text { last } 7 \text { days }\end{array}$ \\
& AND \\
\hline Symptoms: & $\begin{array}{l}\text { At least intermittent pain in the shoulder region without } \\
\text { paresthesias; pain worsened by active elevation } \\
\text { movement of the upper arm as in scratching of the } \\
\text { upper back }\end{array}$ \\
& AND \\
\hline Signs: & $\begin{array}{l}\text { At least one of the following tests positive: } \\
\text { a resisted shoulder abduction, external rotation, or } \\
\text { internal rotation }\end{array}$ \\
& A resisted elbow flexion \\
& a painful arc on active upper arm elevation
\end{tabular}

'Sluiter JK, Rest KM, Frings-Dresen MH. Criteria document for evaluating the work-relatedness of upper-extremity musculoskeletal disorders. Scand J Work Environ Health. 2001;27 Suppl 1:1-102. 


\section{Methods}

- Workers with RCS at baseline

- 3 groups

$\square$ RCS recovery: workers without shoulder symptoms and RCS

$\checkmark$ Shoulder symptoms without clinically diagnosed RCS

$\checkmark$ No recovery RCS: workers with clinically diagnosed RCS 


\section{Statistical methods}

- Associations between recurrence of RCS and

$\checkmark$ Baseline characteristics

- Personal and work-related factors (organizational, biomechanical and psychosocial factors)

- Follow-up characteristics

- Change of physical load, postural load, psychological load and time constraints during the preceding 12 months

- Separately for men and women

- Chi-square, Fisher and Mann-Withney tests 


\section{Results}

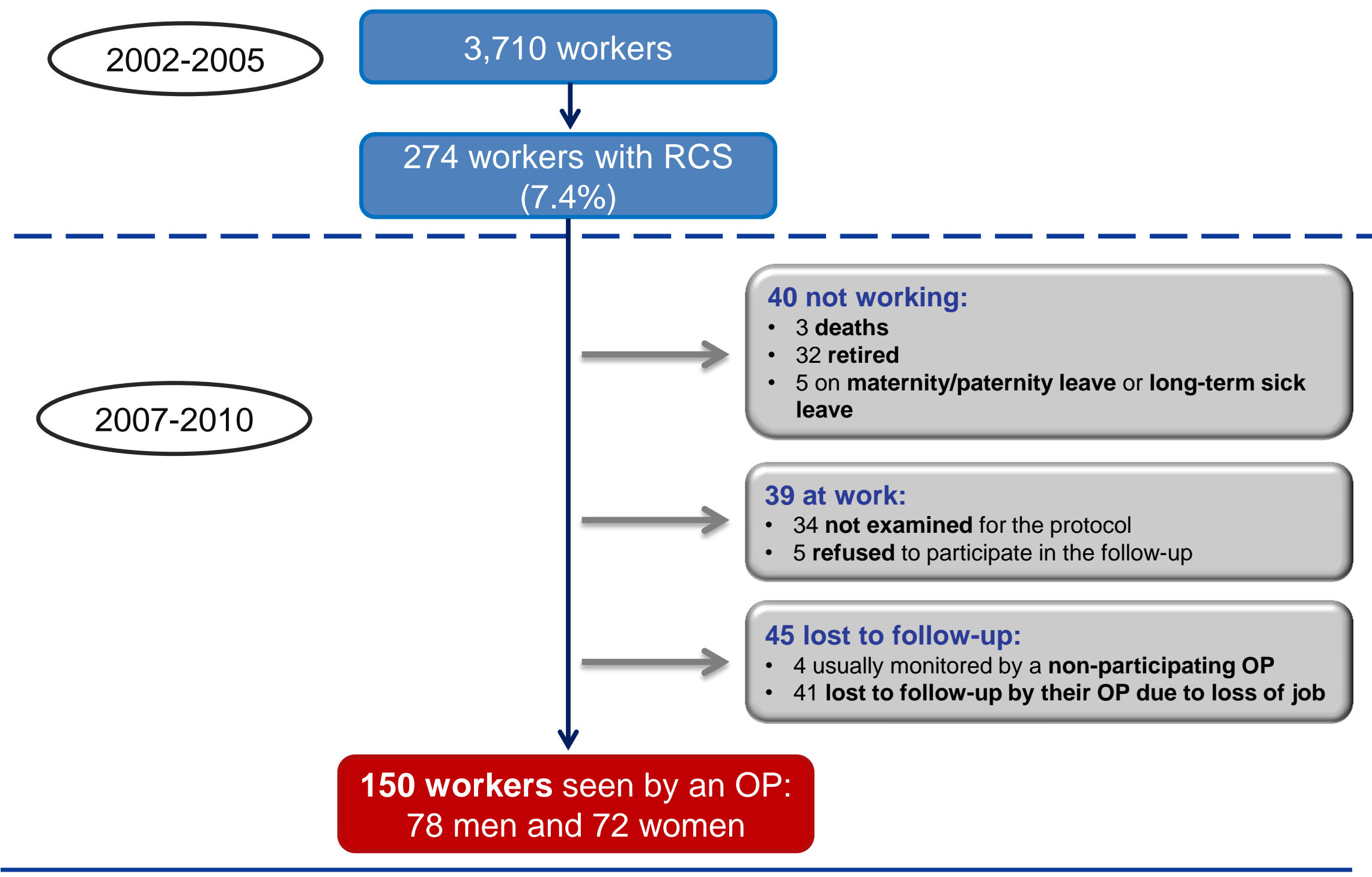




\section{Physical examination at follow-up among workers with RCS in 2002-2005}

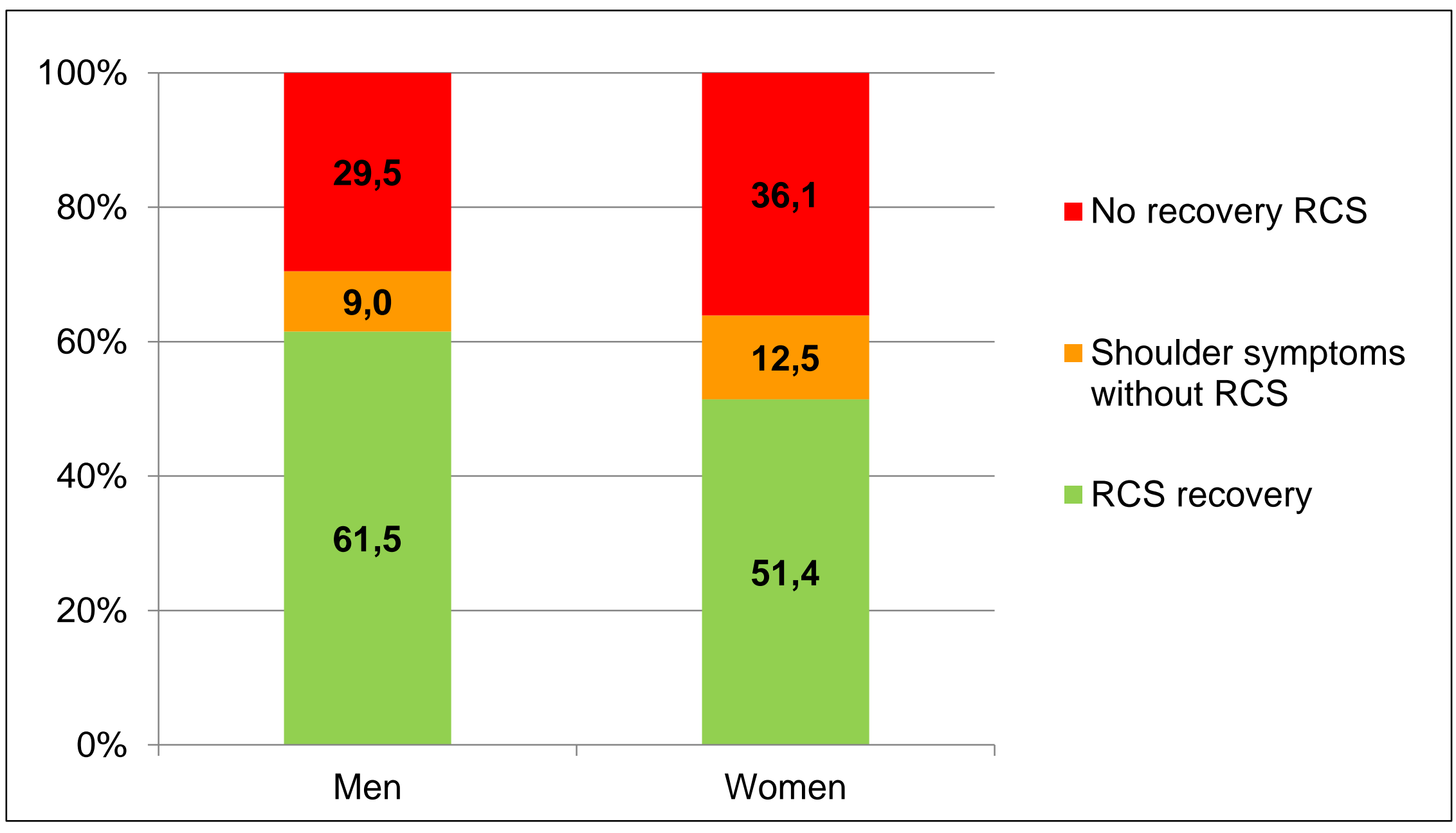




\section{Characteristics at baseline according to health group in men}

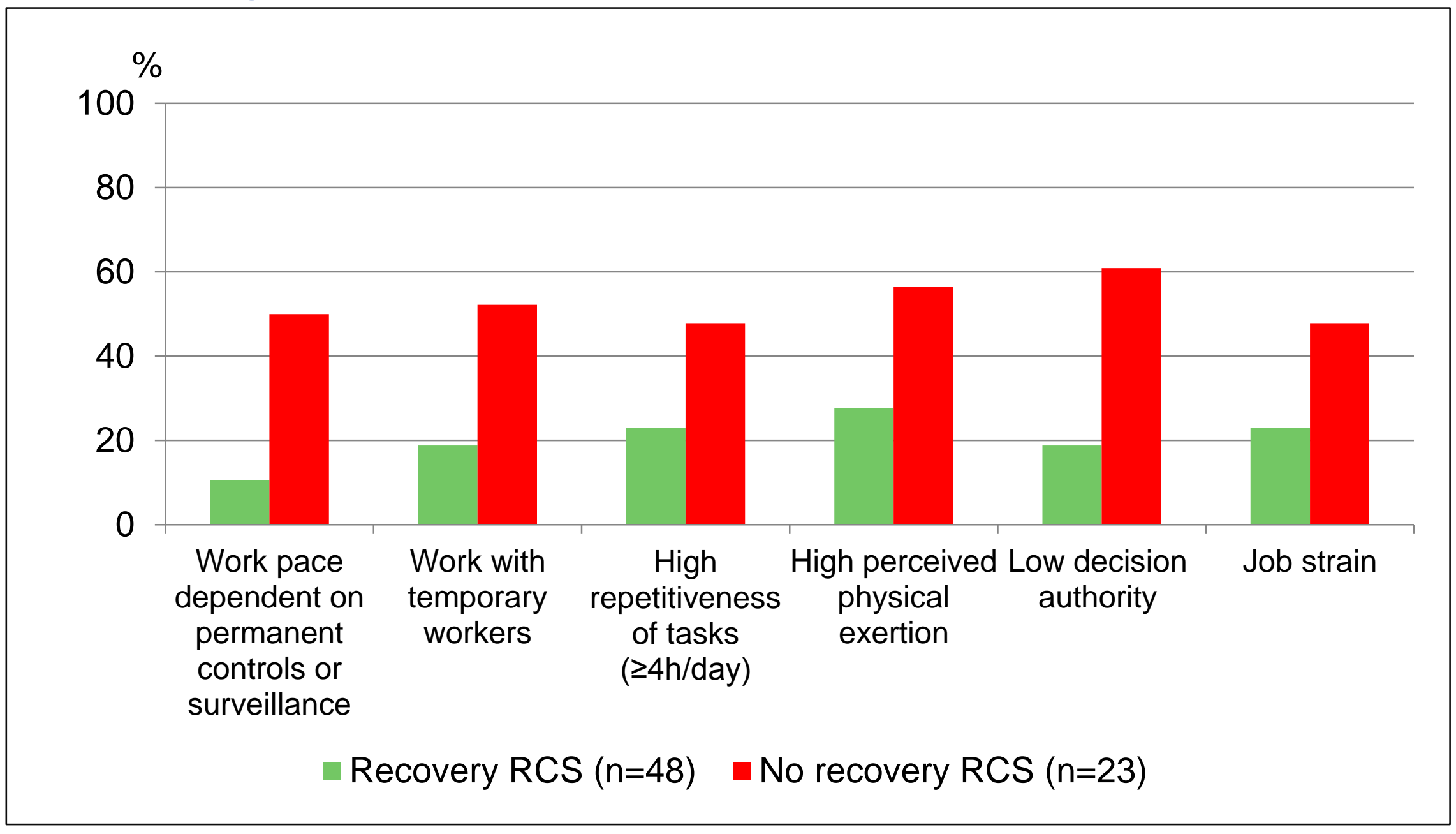




\section{Characteristics at baseline according to health group in women}

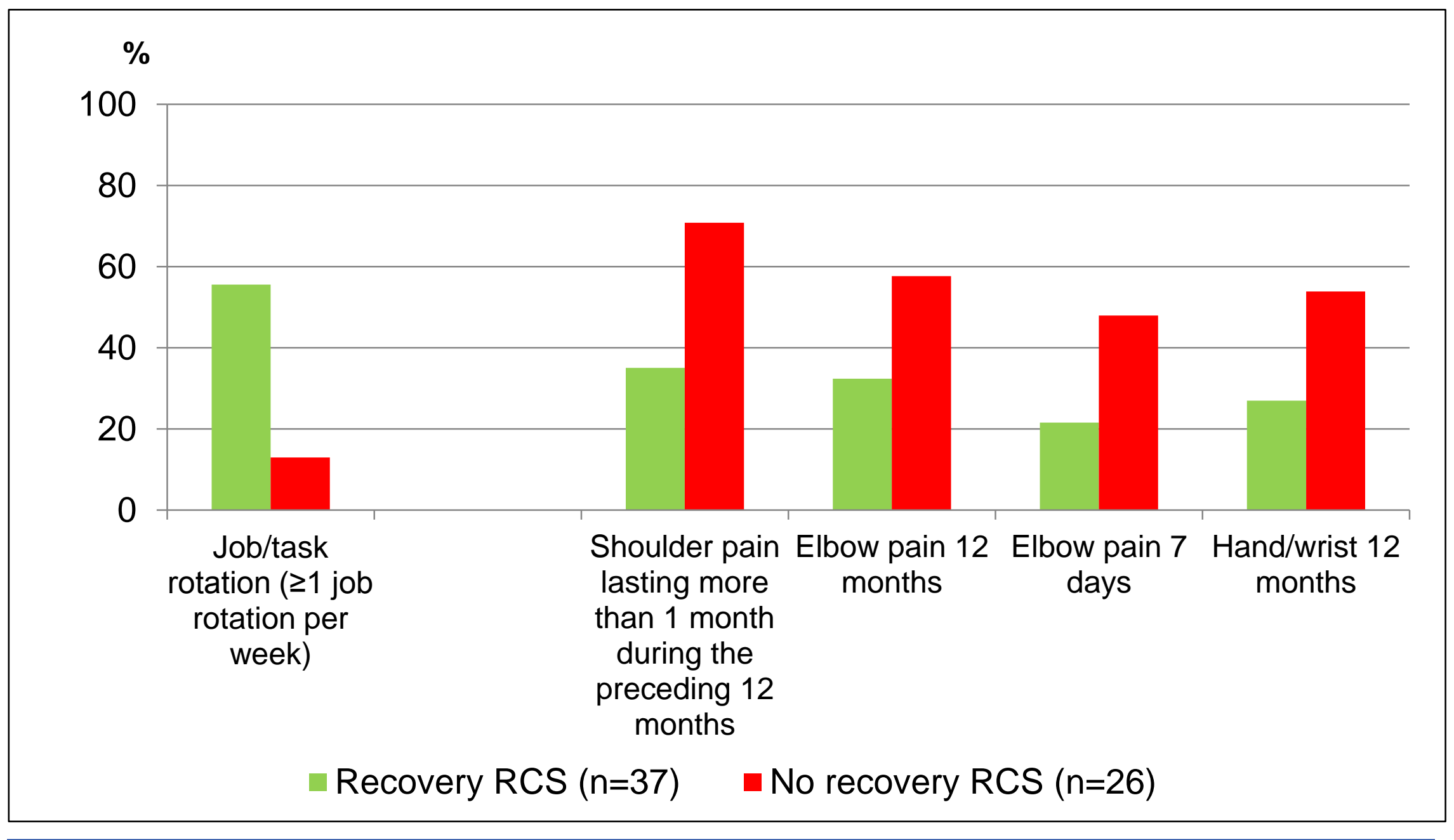




\section{Characteristics at follow-up according to health group \\ - Men}

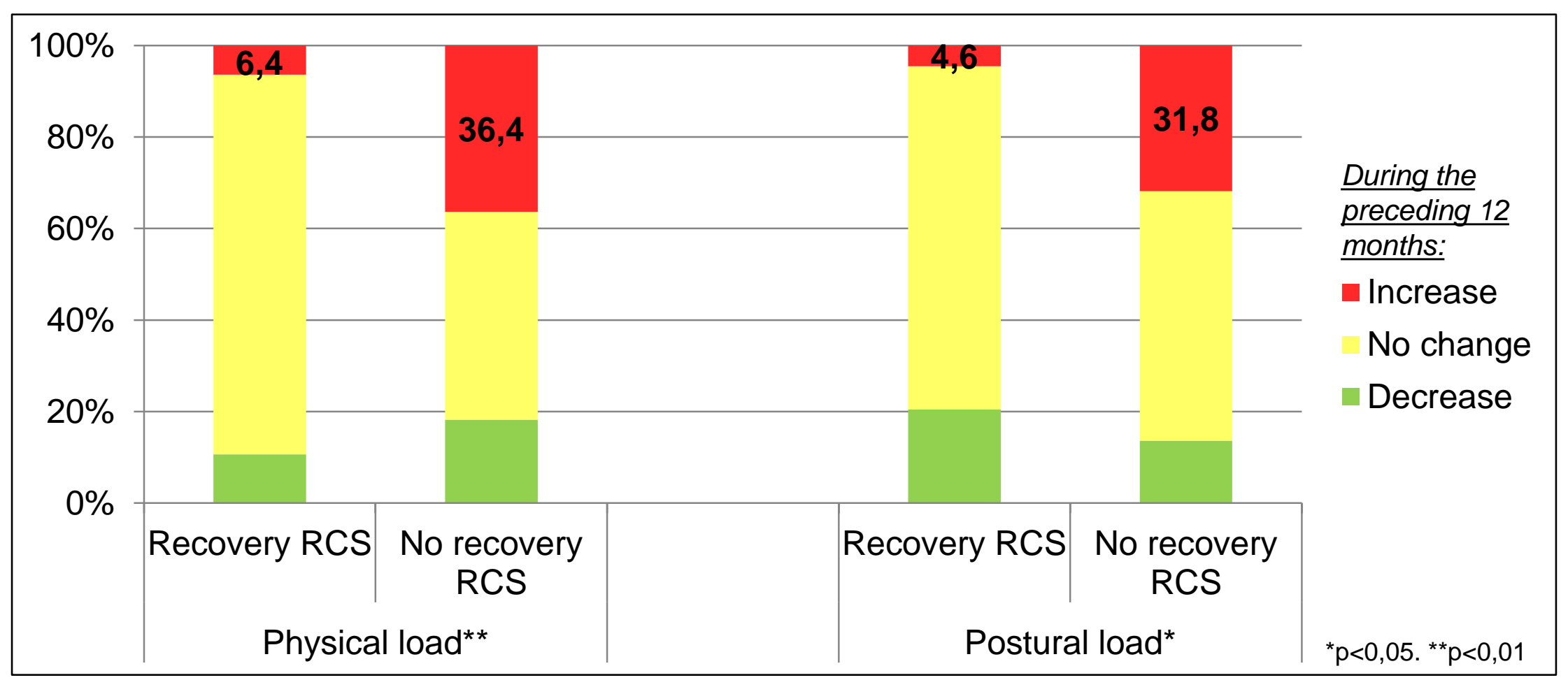

- No differences for psychological load and times constraints

- Women: No differences 


\section{Discussion}

- RCS clinically diagnosed by trained OPS

- Low follow-up rate

口 Only workers still at work considered

- Unemployed people and retired people excluded

- Exposure data self-reported

- Extra-occupational physical activities and individual psychological factors not assessed 


\section{Conclusion}

- High level of recovery from RCS

$62 \%$ for men and $51 \%$ for women

- Factors associated with no recovery:

๑ Men: work-related factors

๑ Women: personal factors

- It would be interesting to act on these factors to prevent them, and to identify workers in whom RCS might become chronic. 


\section{Thank you for your attention}




\section{Working postures and biomechanical constraints}

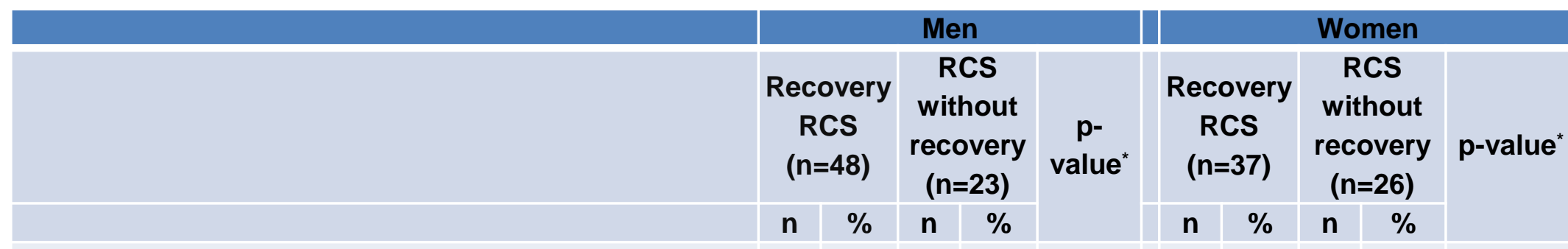

Working postures and biomechanical constraints

\begin{tabular}{|c|c|c|c|c|c|c|c|c|c|c|}
\hline High repetitiveness of tasks ( $\geq 4 \mathrm{~h} /$ day) & 11 & 22.9 & 11 & 47.8 & 0.034 & 17 & 47.2 & 14 & 53.9 & 0.607 \\
\hline High perceived physical exertion ${ }^{\dagger}$ & 13 & 27.7 & 13 & 56.5 & 0.019 & 9 & 24.3 & 5 & 19.2 & 0.632 \\
\hline $\begin{array}{l}\text { Posture with the arms above the shoulder level } \\
(\geq 2 \mathrm{~h} / \text { day })\end{array}$ & 11 & 22.9 & 6 & 26.1 & 0.770 & 6 & 16.2 & 6 & 24.0 & $0.521^{* *}$ \\
\hline Posture with the arms abducted $\left(60^{\circ}\right.$ to $\left.90^{\circ}\right)(\geq 2 \mathrm{~h} /$ day $)$ & 8 & 16.7 & 6 & 26.1 & $0.359^{* *}$ & 8 & 21.6 & 7 & 28.0 & 0.565 \\
\hline Holding hand behind the trunk ( $\geq 2 \mathrm{~h} / \mathrm{day}$ ) & 3 & 6.3 & 2 & 8.7 & $0.656^{* *}$ & 4 & 10.8 & 3 & 12.0 & $1.000^{* *}$ \\
\hline Biomechanical exposure $\ddagger$ & & & & & 0.053 & & & & & 0.565 \\
\hline 0 & 21 & 44.7 & 5 & 21.7 & & 12 & 33.3 & 8 & 32.0 & \\
\hline 1 & 16 & 34.0 & 7 & 30.4 & & 11 & 30.6 & 5 & 20.0 & \\
\hline $2-5$ & 10 & 21.3 & 11 & 47.8 & & 13 & 36.1 & 12 & 48.0 & \\
\hline Use of hand tools ( $\geq 2 \mathrm{~h} /$ day) & 28 & 58.3 & 14 & 60.9 & 0.839 & 17 & 47.2 & 14 & 53.8 & 0.607 \\
\hline Use of vibrating hand tools ( $\geq 2 \mathrm{~h} /$ day) & 11 & 22.9 & 9 & 39.1 & 0.155 & 4 & 10.8 & 0 & 0.0 & $0.136^{* *}$ \\
\hline Pushing or pulling load ( $\geq 2 \mathrm{~h} /$ day) & 11 & 22.9 & 7 & 31.8 & 0.429 & 3 & 8.1 & 5 & 19.2 & $0.257^{\star *}$ \\
\hline Working seated ( $\geq 4 \mathrm{~h} /$ day) & 9 & 18.8 & 3 & 13.0 & $0.739^{* *}$ & 21 & 56.8 & 9 & 34.6 & 0.083 \\
\hline
\end{tabular}

RCS: rotator cuff syndrome.

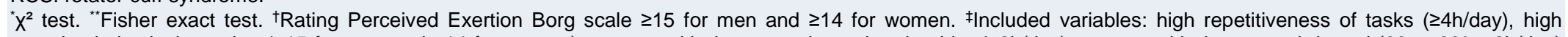

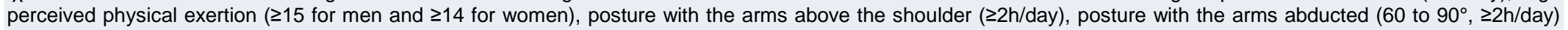
and holding hand behind the trunk ( $\geq 2 \mathrm{~h} /$ day) 


\section{Work characteristics at follow-up according to}

health group in men

\begin{tabular}{|c|c|c|c|c|c|}
\hline & \multicolumn{2}{|c|}{$\begin{array}{c}\text { Recovery } \\
\text { RCS (n=48) }\end{array}$} & \multicolumn{2}{|c|}{$\begin{array}{l}\text { No recovery } \\
\operatorname{RCS}(n=23)\end{array}$} & \multirow[t]{2}{*}{$\mathbf{p}$} \\
\hline & $\mathbf{n}$ & $\%$ & $\mathbf{n}$ & $\%$ & \\
\hline Job change in the same company & 13 & 27.1 & 5 & 22.7 & 0.699 \\
\hline Company change & 4 & 8.3 & 5 & 22.7 & 0.128 \\
\hline
\end{tabular}

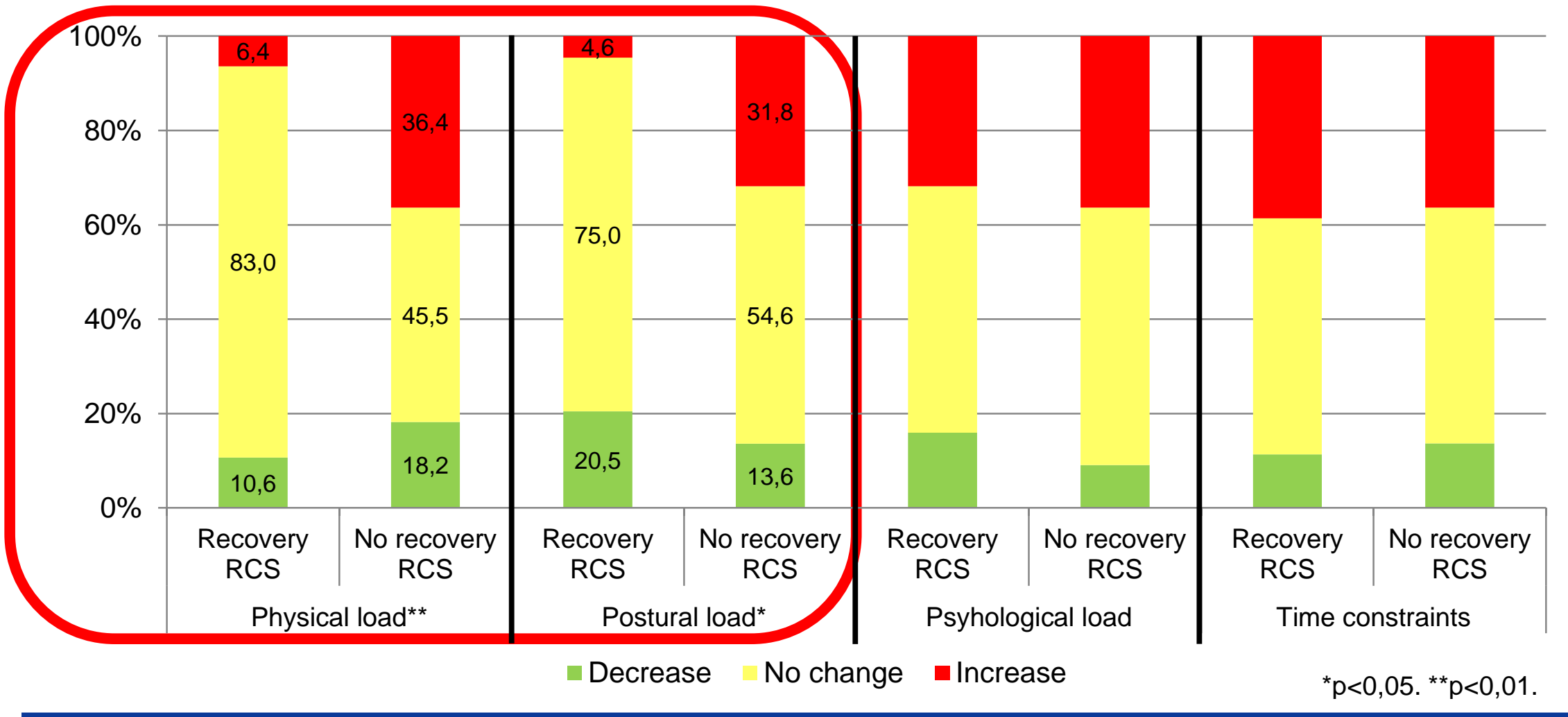




\section{Work characteristics at follow-up according to health group in women}

\begin{tabular}{|c|c|c|c|c|c|}
\hline & \multicolumn{2}{|c|}{$\begin{array}{c}\text { Recovery } \\
\text { RCS ( } n=37)\end{array}$} & \multicolumn{2}{|c|}{$\begin{array}{l}\text { No recovery } \\
\text { RCS }(n=26)\end{array}$} & \multirow[t]{2}{*}{$\mathbf{p}$} \\
\hline & $\mathbf{n}$ & $\%$ & $\mathbf{n}$ & $\%$ & \\
\hline Job change in the same company & 7 & 18.9 & 9 & 34.6 & 0.159 \\
\hline Company change & 6 & 16.2 & 0 & 0.0 & 0.038 \\
\hline
\end{tabular}

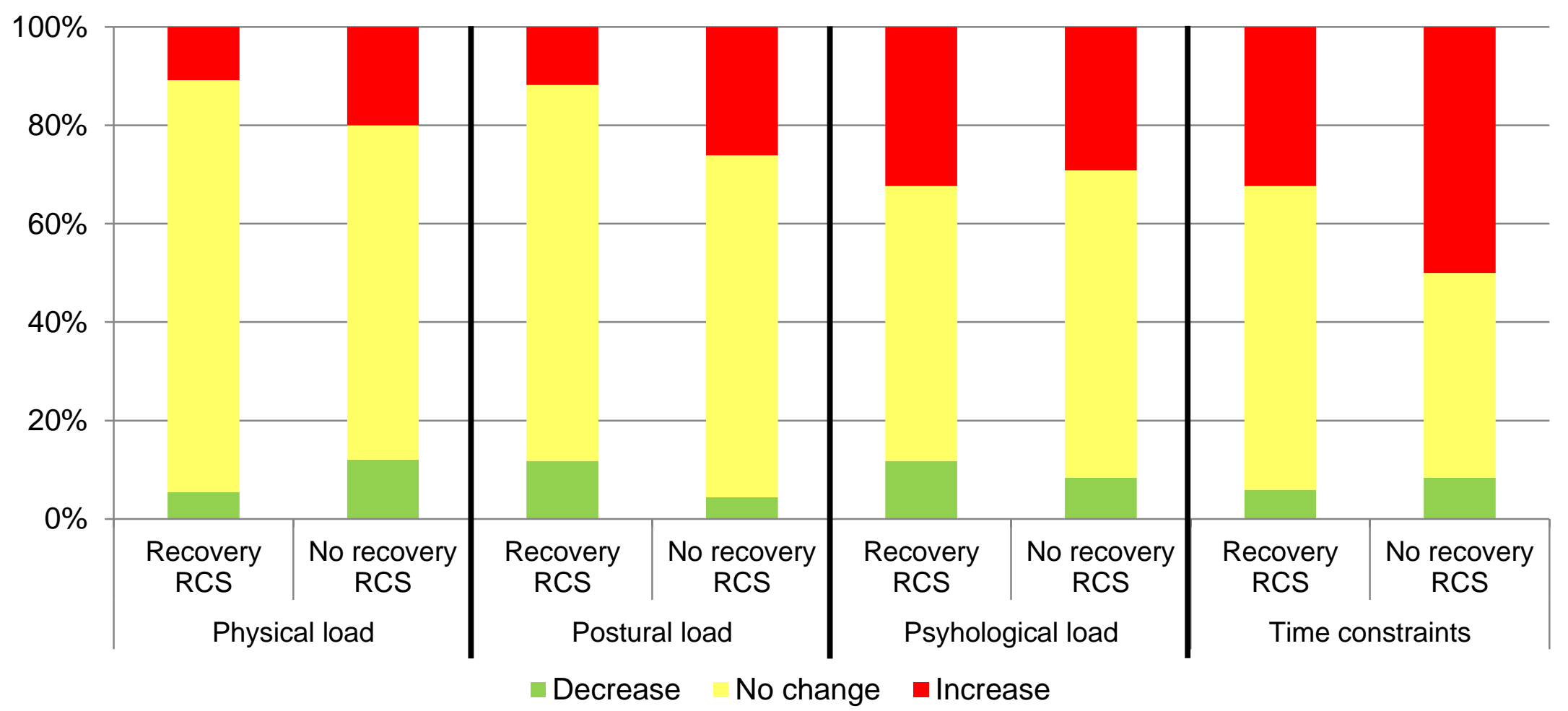


Nom du test

\section{Photo}

Type de test

\section{1}

Mouvement actif de circumduction de l'épaule, pour le syndrome de la coiffe des rotateurs dans le plan de l'omoplate

Position de départ du Debout avec les bras pendants, les pouces dirigés vers l'avant

\section{sujet}

Position de départ de l'examinateur

Consigne donnée au sujet

Positif si
Debout, face au sujet, il positionne les membres supérieurs du sujet à $30^{\circ}$ en avant du plan frontal

«Levez les bras jusqu'à hauteur des épaules, tournez les paumes vers le haut et levez les bras jusqu'à ce que vos mains se touchent au-dessus de la tête »

Douleur au cours du mouvement (entre 60 et $120^{\circ}$ d'abduction)

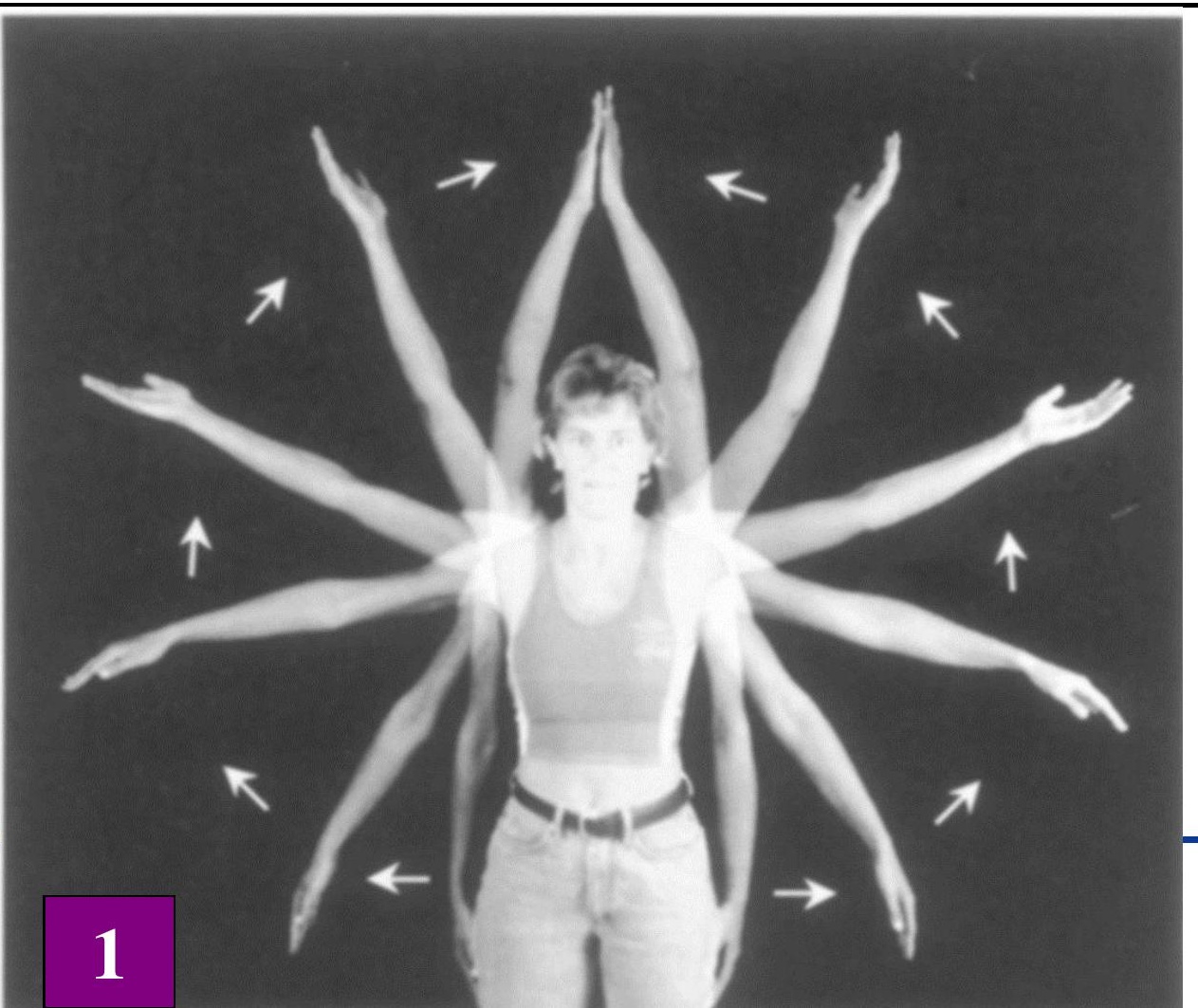




\section{1. abduction / rotation externe}

Comparaison D / G

\section{Photo}

Type de test

Position de départ Debout

du sujet

Position de départ Debout, derrière le sujet

de l'examinateur

Consigne donnée au sujet

Positif si

\section{2} des doigts »
Mouvement actif de l'épaule, pour le syndrome de la coiffe des rotateurs

«Placez votre main derrière la tête et essayez de toucher le haut de l'omoplate du bout

Douleur locale de l'épaule au cours ou à la fin du mouvement 
Test de l'élévation active de l'épaule

2. abduction / rotation interne

\section{Comparaison D / G}

Photo

Type de test

Position de départ

du sujet

Position de départ Debout, derrière le sujet de l'examinateur

Consigne donnée au sujet

Positif si

\section{3}

Debout
Mouvement actif de l'épaule pour le syndrome de la coiffe des rotateurs

«Placez votre main derrière le dos et essayez de toucher le bas de votre autre omoplate du bout des doigts »

Douleur locale de l'épaule au cours ou à la fin du mouvement 


\section{Adduction \\ Comparaison D / G}

\section{Photo}

Type de test

Position de départ du sujet

Position de départ de

l'examinateur

Consigne donnée au sujet

Positif si
4

Mouvement actif de l'épaule pour le syndrome de la coiffe des rotateurs

Debout

Debout, face au sujet

«Empaumez le sommet de votre autre épaule»

Douleur locale de l'épaule au cours ou à la fin du mouvement

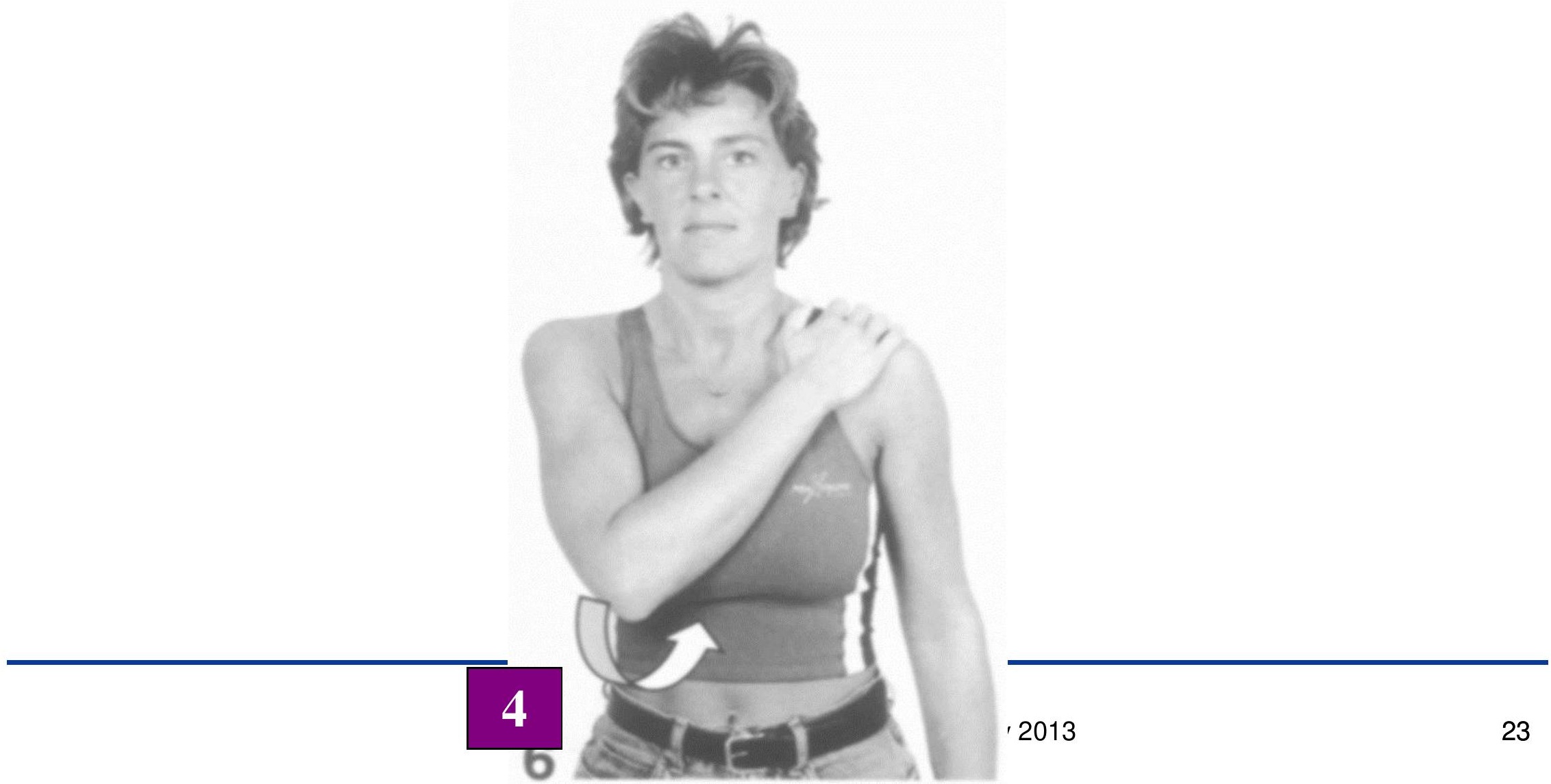




\section{Photo}

Type de test

Position de départ du sujet

Position de départ de l'examinateur Consigne donnée au sujet

Positif si
5

Résistance isométrique pour le syndrome de la coiffe des rotateurs

Assis, avec le bras D en abduction de $10-20^{\circ}$

Debout à $\mathrm{D}$ du sujet. La main $\mathrm{G}$ stabilise le sommet de l'épaule, la main $\mathrm{D}$ appuie pour s'opposer à l'abduction du bras.

«Maintenez le bras dans cette position et résistez contre ma force »

Douleur locale de l'épaule (muscle supra-épineux / sus-épineux)

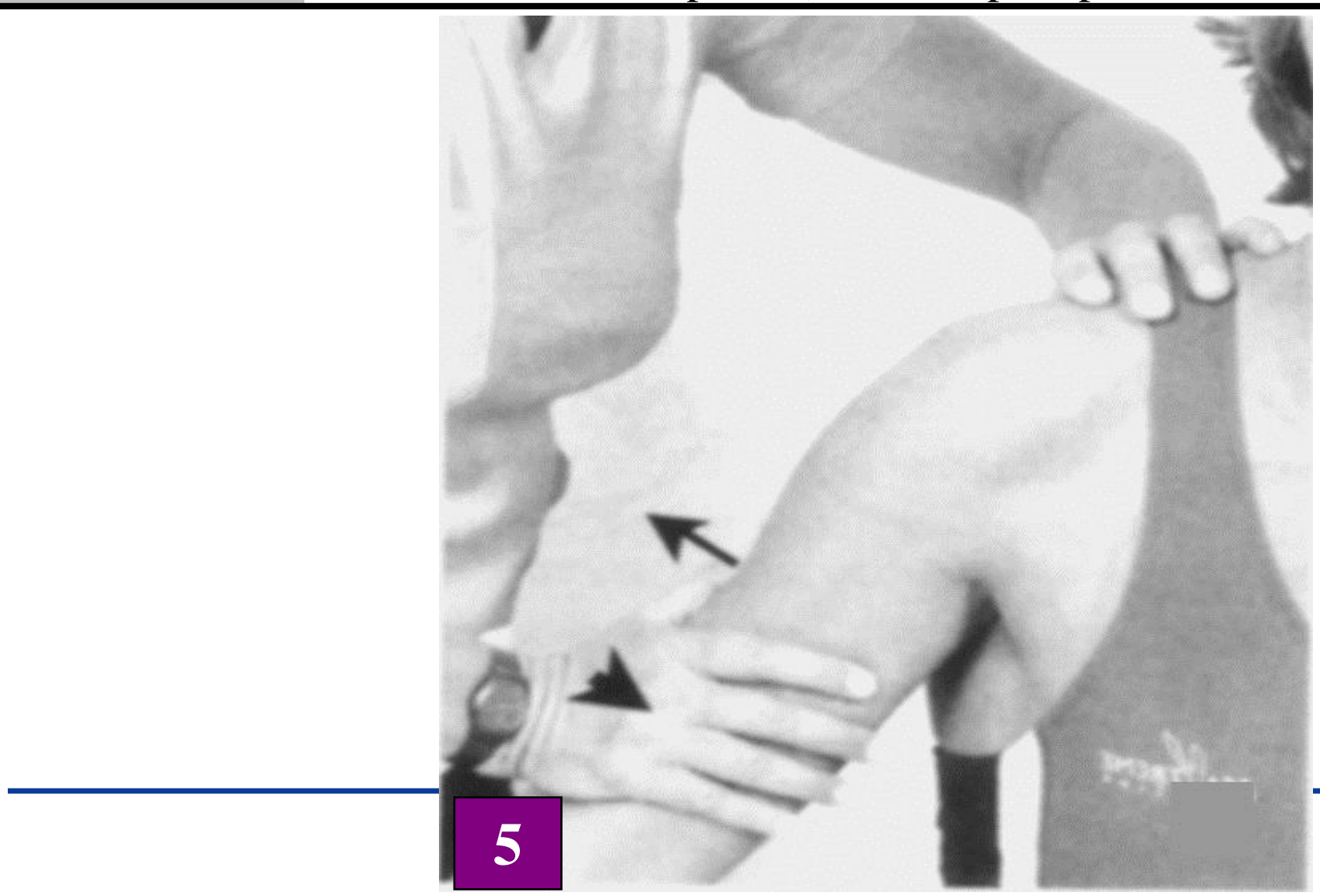




\section{Photo}

Type de test

Position de départ du sujet

Position de départ de l'examinateur et réalisation du

test

Consigne donnée au sujet

Positif si

\section{6}

Résistance isométrique pour le syndrome de la coiffe des rotateurs

Assis, bras $\mathrm{D}$ contre le corps, coude $\mathrm{D}$ fléchi à $90^{\circ}$, poignet $\mathrm{D}$ en position neutre

Debout à $\mathrm{D}$ du sujet. La main $\mathrm{G}$ contrôle la position du coude, la main $\mathrm{D}$ empaume la face dorsale de l'avant-bras D et appuie pour entraîner l'épaule en rotation interne

«Maintenez le coude contre votre corps et résistez contre ma force »

Douleur locale de l'épaule (muscle infra-épineux / sous-épineux)

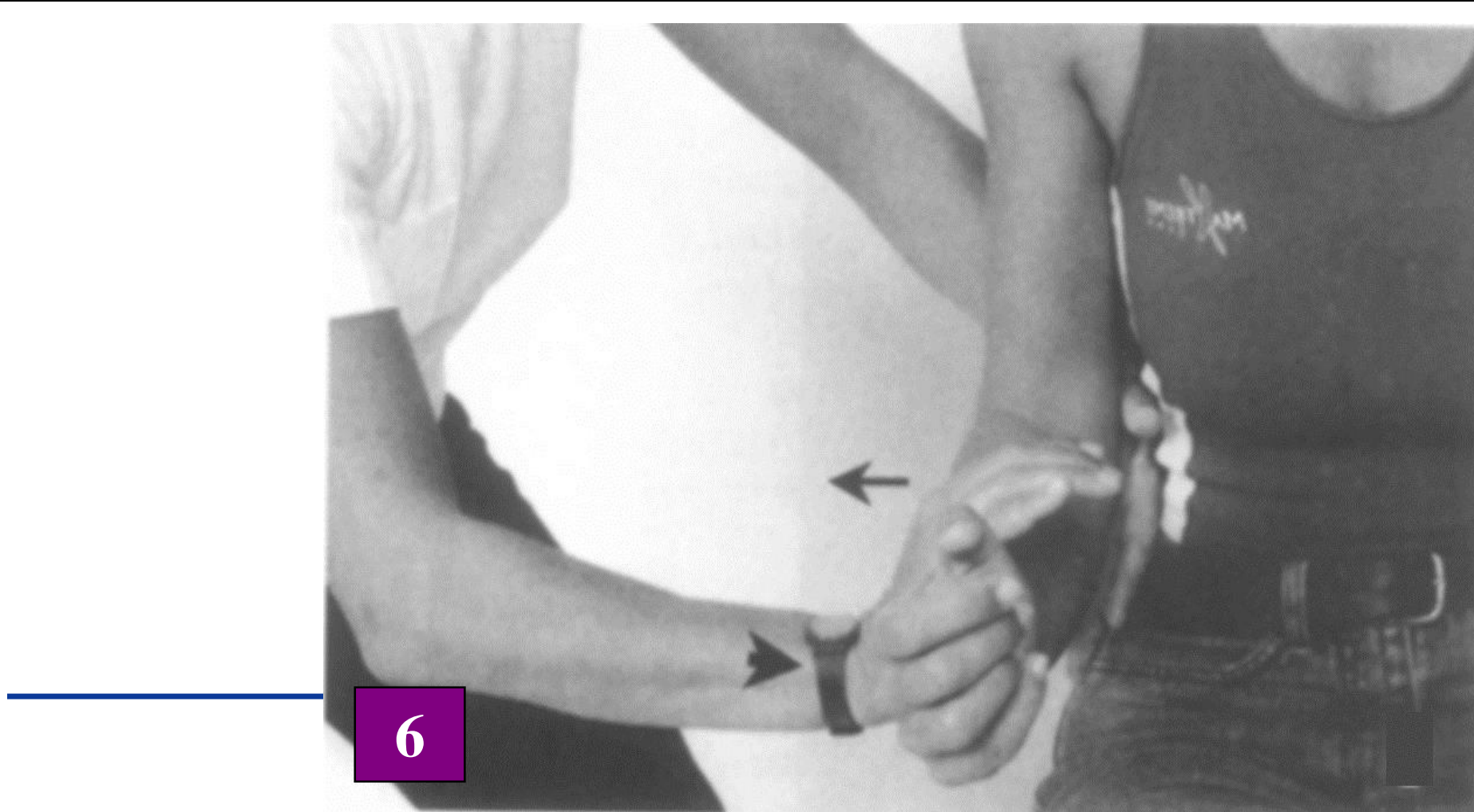




\section{Photo}

Type de test

Position de départ du sujet Position de départ de l'examinateur

Consigne donnée au sujet

Positif si
7

Résistance isométrique pour le syndrome de la coiffe des rotateurs Assis, bras $\mathrm{D}$ contre le corps, coude $\mathrm{D}$ fléchi à $90^{\circ}$, poignet $\mathrm{D}$ en position neutre

Debout, face au sujet. La main G contrôle la position du coude, la main D empaume la face ventrale de l'avant-bras et appuie pour empêcher la rotation interne de l'épaule.

«Maintenez le coude contre votre corps et résistez contre ma force »

Douleur locale de l'épaule (muscle sub-scapulaire / sous-scapulaire)

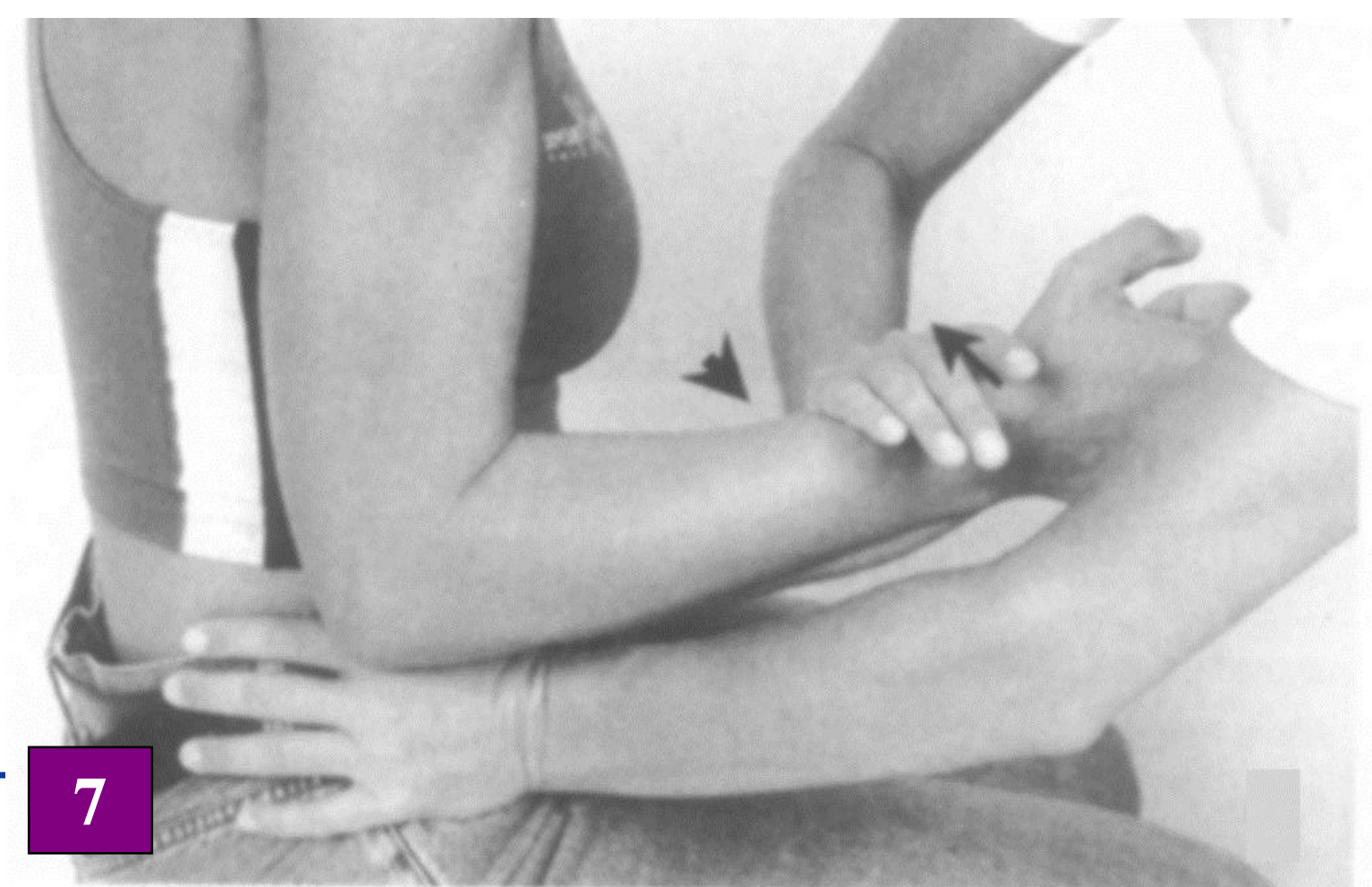




\section{Photo}

Type de test

Position de départ du sujet

Position de départ de l'examinateur et réalisation du

test

Consigne donnée au sujet

Positif si
8

Résistance isométrique pour le syndrome de la coiffe des rotateurs

Assis, bras D à $90^{\circ}$ d'antéflexion, avant-bras D en supination (paume vers le haut), coude $D$ légèrement fléchi

Debout à $\mathrm{G}$ du sujet. La main $\mathrm{D}$ stabilise l'articulation gléno-humérale, la main $\mathrm{G}$ empaume la face ventrale de l'avant-bras D et appuie pour étendre le coude

«Maintenez le bras dans cette position et résistez contre ma force »

Douleur locale au-dessus de l'insertion du tendon du biceps brachial

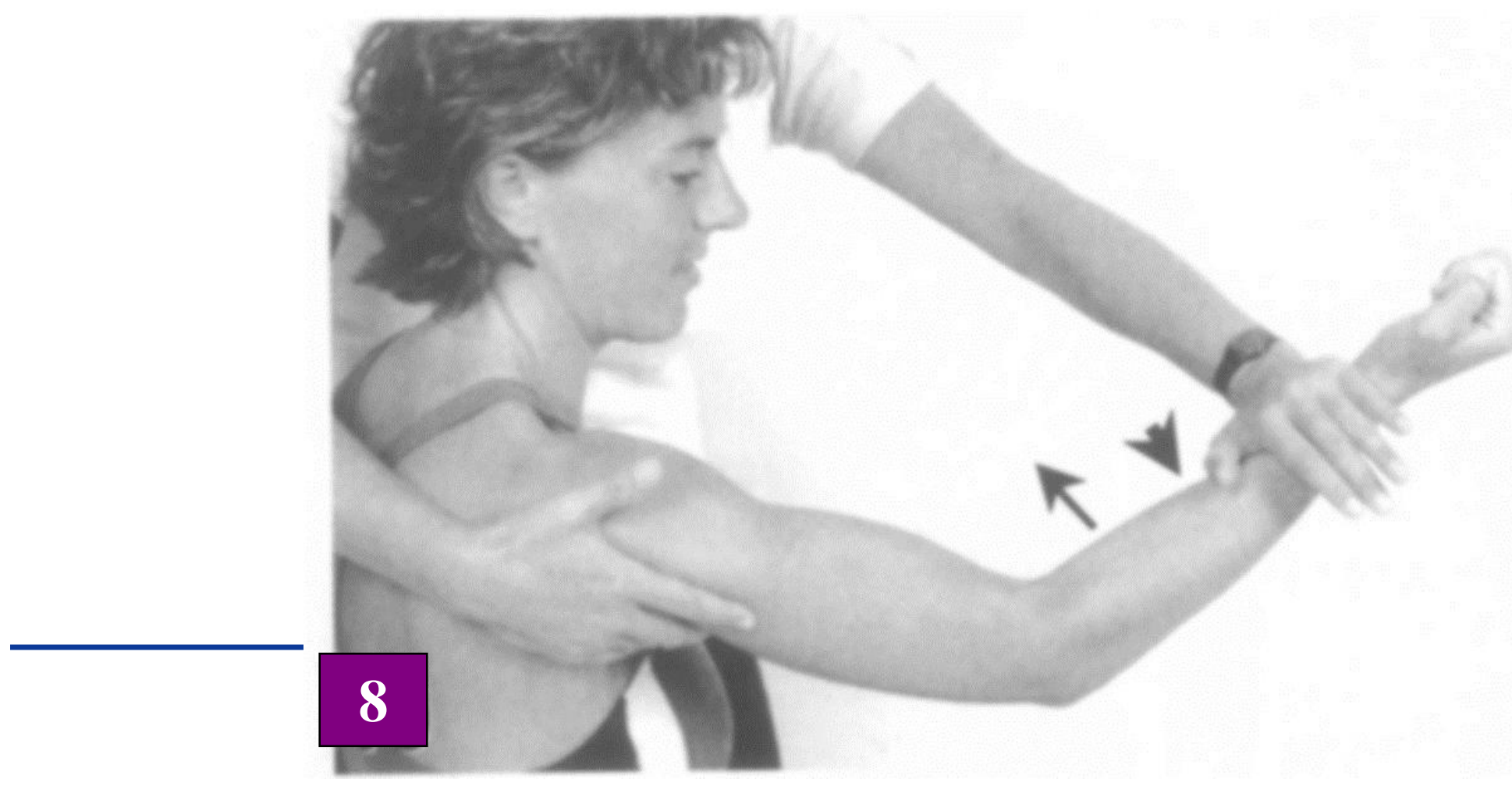

